\title{
T-duality for circle bundles via noncommutative geometry
}

\author{
VArghese Mathai And Jonathan RosenberG
}

\begin{abstract}
Recently Baraglia showed how topological T-duality can be extended to apply not only to principal circle bundles, but also to non-principal circle bundles. We show that his results can also be recovered via two other methods: the homotopy-theoretic approach of Bunke and Schick, and the noncommutative geometry approach which we previously used for principal torus bundles. This work has several interesting byproducts, including a study of the $K$ theory of crossed products by $\widetilde{O}(2)=\operatorname{Isom}(\mathbb{R})$, the universal cover of $O(2)$, and some interesting facts about equivariant $K$-theory for $\mathbb{Z} / 2$. In the final section of this paper, some of these results are extended to the case of bundles with singular fibers, or in other words, non-free $O(2)$-actions.
\end{abstract}

\section{Introduction}

T-duality in physics (the "T" standing for "target space") is a duality between two string theories, that interchanges "winding" and "momentum" modes and replaces tori in the spacetime target space by their duals. (Duality here means a symmetry that relates theories that mathematically look different but that predict the same physics.) Topological T-duality, which began from [1] and [6,7], aims to capture the basic topological content in T-duality. It has expanded to a vast literature, but for a general survey, one can see the book [28]. Roughly speaking, there are three main approaches to topological T-duality: via homotopy theory (as in [8]), via noncommutative geometry (as in [20]), and via stacks, groupoids, and/or equivariant topology (as in $[9,13,22]$ ). In the usual setup of topological T-duality, one is given a principal torus bundle $E \stackrel{p}{\rightarrow} X$ together with a class $h \in H^{3}(E, \mathbb{Z})$, and aims (maybe after imposing other conditions) to produce from this data a "dual bundle" $E^{\#} \stackrel{p^{\#}}{\rightarrow} X$ and a dual class $h^{\#} \in H^{3}(E, \mathbb{Z})$. From the point of view of the original situation in physics, $E$ here is spacetime for a type II superstring theory, $h$ is the H-flux (the cohomology class of the field strength 
of the Neveu-Schwarz B-field), and we are suppressing non-topological data such as the actual B-field and the metric.

Recently, David Baraglia [2, 4] showed that this setup can be generalized to the case where $E \stackrel{p}{\rightarrow} X$ is a non-principal circle bundle, and even [3] extended many of his results to affine torus bundles of arbitrary rank.

Our aim in this paper is to give new approaches to Baraglia's results on circle bundles, that show how they also fit into the topological framework initiated by Bunke and Schick in [8] and the noncommutative geometry approach which we used in $[20,21]$. This grew out of a larger project, still in progress, of trying to identify a good larger category of "generalized bundles" that incorporates the bundles of $[14,15]$ and is well-adapted to the noncommutative geometry approach to topological T-duality.

We begin with a few definitions.

Definition 1.1. A principal circle bundle over a space $X$ is a map $E \stackrel{p}{\rightarrow} X$, where $E$ is a space equipped with a free action of $\mathbb{T}$ (the unit circle in the complex plane, with its group structure coming from complex multiplication) for which $p$ is the projection onto the orbit space $E / \mathbb{T}$ and the map is locally trivial, in the sense that for any $x \in X$, there is an open set $U$ containing $x$ for which one has a commuting diagram

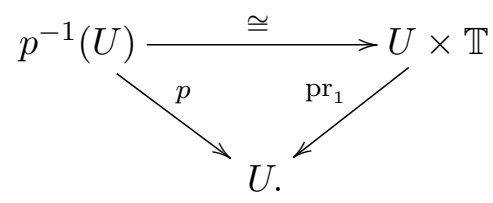

A non-principal circle bundle over $X$, on the other hand, is a topological fiber bundle over $X$ with fiber $S^{1}$ and structure group Homeo $\left(S^{1}\right)$. Since the inclusions $O(2)=\operatorname{Isom}\left(S^{1}\right) \hookrightarrow \operatorname{Diff}\left(S^{1}\right) \hookrightarrow \operatorname{Homeo}\left(S^{1}\right)$ are homotopy equivalences, as observed in [2], there is no loss of generality in assuming that the bundle has structure group $O(2)$. Thus the difference between principal and non-principal circle bundles simply amounts to replacing $S O(2)$ (which can be identified with $\mathbb{T}=U(1)$ in the usual way) by $O(2)$. It is convenient to view $O(2)$ as $S O(2) \rtimes\{1, j\}$, where $j$ is the reflection matrix

$$
\left(\begin{array}{cc}
1 & 0 \\
0 & -1
\end{array}\right)
$$

Thus for reasonable spaces $X$ (in applications, $X$ will always be a manifold of finite homotopy type), a non-principal circle bundle over $X$ is classified by a map $\varphi: X \rightarrow B O(2)$ up to homotopy. This in turn gives rise to a principal 
$O(2)$-bundle $\pi: \widetilde{E} \rightarrow X$ and thus a commuting diagram

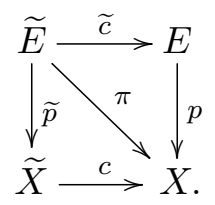

Here $E \stackrel{p}{\rightarrow} X$ is the original non-principal circle bundle, $\widetilde{c}$ and $c$ are two-fold covering maps, and $\widetilde{E} \stackrel{\widetilde{p}}{\rightarrow} \widetilde{X}$ is a principal circle bundle gotten by taking the orbit space of $\widetilde{E}$ under the subgroup $S O(2)$ of $O(2)$. The covering maps $\widetilde{c}$ and $c$ come from dividing out by the action of $j$. The covering $\widetilde{X} \stackrel{c}{\rightarrow} X$ is classified by an element $\xi=w_{1}(p) \in H^{1}\left(X, \mathbb{F}_{2}\right)\left(\mathbb{F}_{2}\right.$ denoting the field of two elements), or equivalently, by the homotopy class of the composite $X \stackrel{\varphi}{\rightarrow}$ $B O(2) \stackrel{B q}{\longrightarrow} B \mathbb{Z} / 2 \simeq K(\mathbb{Z} / 2,1)$, where $B q$ is the map on classifying spaces induced by the quotient map of topological groups $q: O(2) \rightarrow \mathbb{Z} / 2$. Then the isomorphism class of the bundle $p$ is determined by the combination of $\xi \in H^{1}\left(X ; \mathbb{F}_{2}\right)$ and $c_{1}(\widetilde{p}) \in H^{2}(\widetilde{X}, \mathbb{Z})$, which we can also view as living in $H^{2}\left(X, \mathbb{Z}_{\xi}\right)$, where $\mathbb{Z}_{\xi}$ is the local coefficient system on $X$ locally isomorphic to $\mathbb{Z}$ and determined by $\xi$. We call this the Chern class $c_{1}(p)$.

Remark 1.2. As with principal $S^{1}$ bundles, a non-principal circle bundle $E \stackrel{p}{\rightarrow} X$ comes with a Gysin sequence, that arises from the fact that the Leray-Serre spectral sequence for computing the cohomology of the total space of the fibration $S^{1} \rightarrow E \stackrel{p}{\rightarrow} X$ has only two rows and thus collapses at $E_{3}$. However, as the fundamental group of the base $X$ acts trivially on $H^{0}$ of the fiber but nontrivially on $H^{1}\left(S^{1}\right) \cong \mathbb{Z}$, the spectral sequence has $E_{2}^{p, 0}=$ $H^{p}(X, \mathbb{Z})$ but $E_{2}^{p, 1}=H^{p}\left(X, \mathbb{Z}_{\xi}\right)$, the cohomology of $X$ with local coefficients. Recall that $\mathbb{Z}_{\xi} \otimes \mathbb{Z}_{\xi} \cong \mathbb{Z}$ as local coefficient systems, so the cup product of two twisted cohomology classes lives back in untwisted cohomology. The Gysin sequence thus takes the form

$$
\begin{aligned}
\cdots \rightarrow H^{p}\left(X, \mathbb{Z}_{\xi}\right) \stackrel{\cup c_{1}(p)}{\longrightarrow} H^{p+2}(X, \mathbb{Z}) & \stackrel{p^{*}}{\longrightarrow} H^{p+2}(E, \mathbb{Z}) \\
& \stackrel{p_{!}}{\longrightarrow} H^{p+1}\left(X, \mathbb{Z}_{\xi}\right) \stackrel{\cup c_{1}(p)}{\longrightarrow} \cdots .
\end{aligned}
$$

(Note the alternation between twisted and untwisted cohomology.)

With these preliminaries, we can now state one of Baraglia's main results:

Theorem 1.3 (Baraglia [2, Proposition 4.1]). For any circle bundle $E \stackrel{p}{\rightarrow} X$ as above, with invariants $\xi=w_{1}(p) \in H^{1}\left(X, \mathbb{F}_{2}\right)$ and $c_{1}(p) \in$ 
$H^{2}\left(X, \mathbb{Z}_{\xi}\right)$, and for any choice of "H-flux" $h \in H^{3}(E, \mathbb{Z})$, there is a unique $T$-dual circle bundle $E^{\#} \stackrel{p^{\#}}{\longrightarrow} X$ and there is a unique T-dual $H$-flux $h^{\#} \in$ $H^{3}(E, \mathbb{Z})$, characterized by the following axioms:

(1) T-duality is natural, so the T-dual of a pull-back is the pull-back of the dual.

(2) T-duality is involutive, so that $(E, p, h)$ is the T-dual of $\left(E^{\#}, p^{\#}, h^{\#}\right)$.

(3) $\xi=w_{1}(p)=w_{1}\left(p^{\#}\right)$, so the double covering of $X$ defined by the T-dual is the same as $X \stackrel{c}{\rightarrow} X$ defined by $p$.

(4) $p_{!}(h)=c_{1}\left(p^{\#}\right)$ and $\left(p^{\#}\right) !\left(h^{\#}\right)=c_{1}(p)$.

(5) $h$ and $h^{\#}$ agree after pull-back to the "Poincaré bundle" $E \times_{X} E^{\#}$ $\rightarrow X$.

In Section 2 below, we will show how to give another proof of Theorem 1.3 following the outline of the method in [8]. In Section 3, we will give still another proof using $C^{*}$-algebra crossed products, which also leads to the matching of twisted $K$-groups for $(p, h)$ and $\left(p^{\#}, h^{\#}\right)$ as found by Baraglia in [2, Proposition 6.1].

Our method of proof uses an analogue of Connes' "Thom Isomorphism Theorem" [10] for computing $K$-theory for the crossed product of a $C^{*}$ algebra by an action of $\widetilde{O}(2)=\mathbb{R} \rtimes\{1, j\}$. The proof of this theorem, which uses a construction in equivariant $K$-theory from [29], occupies Section 4 . Finally, Section 5 deals with extension of the main results to the case of $O(2)$ bundles with singular fibers, that is $O(2)$-actions that are not necessarily free, following a method from [22].

\section{A homotopy-theoretic construction}

The following definition is basically copied from [8, Definition 2.1].

Definition 2.1. Let $X$ be a topological space having the homotopy type of a CW complex. A pair over $X$ will consist of a (non-principal) circle bundle $E \stackrel{p}{\rightarrow} X$ in the sense of Definition 1.1, together with a class $h \in H^{3}(E, \mathbb{Z})$. Two pairs $(E \stackrel{p}{\rightarrow} X, h)$ and $\left(E^{\prime} \stackrel{p^{\prime}}{\rightarrow} X, h^{\prime}\right)$ are said to be isomorphic if there is a bundle isomorphism $\varphi$ making the diagram

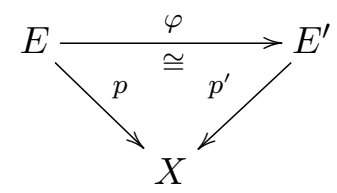


commute and satisfying $\varphi^{*}\left(h^{\prime}\right)=h$. Let $\mathcal{P}(X)$ denote the set of isomorphism classes of pairs over $X$. It is obvious that the map $X \mapsto \mathcal{P}(X)$ is a homotopyinvariant functor on the category of spaces with the homotopy type of a CW complex, with values in pointed sets. $(\mathcal{P}(X)$ has no special structure other than that of a set, but it does has a distinguished basepoint, namely the class of the trivial pair $\left(X \times S^{1} \stackrel{\mathrm{pr}_{1}}{\longrightarrow} X, 0\right)$.)

The following result is then a slight modification of [8, Proposition 2.6].

Theorem 2.2. The functor $X \mapsto \mathcal{P}(X)$ is a representable functor on the homotopy category of spaces with the homotopy type of a $C W$ complex. More precisely, there is a $C W$ complex $R$, unique up to homotopy equivalence, with the following properties:

(1) There is a canonical pair $(E \stackrel{p}{\rightarrow} R, h)$ over $R$.

(2) For any space $X$ with the homotopy type of a $C W$ complex, every pair over $X$ is pulled back from the canonical pair via some map $X \rightarrow R$.

(3) Two pairs over $X$ are isomorphic if and only if their classifying maps $X \rightarrow R$ are homotopic (note that $X$ need not be pointed, so this is unbased homotopy).

Furthermore, we can make the homotopy type of $R$ precise. The space $R$ is connected, and its universal cover $\widetilde{R}$ is the classifying space in [8, Definition 2.4], namely a two-stage Postnikov system

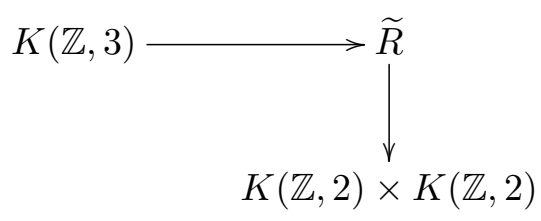

with $k$-invariant $k \in H^{4}(K(\mathbb{Z}, 2) \times K(\mathbb{Z}, 2), \mathbb{Z})$ given by the cup product of the canonical classes in the two $H^{2}(\mathbb{Z}, 2)$ factors.

However, the space $R$ has $\pi_{1}(R) \cong \mathbb{Z} / 2$, and $R$ is not simple; $\pi_{1}(R)$ acts non-trivially on $\pi_{2}(R) \cong \mathbb{Z}^{2}$, with the generator of $\pi_{1}(R)$ acting on $\pi_{2}(R) \cong$ $\mathbb{Z}^{2}$ by -1 .

Proof. We begin by constructing the space $R$. Recall that the classifying space of the orthogonal group $O(2)$ comes with a fibration $B S O(2) \rightarrow$ $B O(2) \rightarrow B(\mathbb{Z} / 2)$, and since the quotient group $\mathbb{Z} / 2$ acts non-trivially on $S O(2)$, this fibration is nontrivial; in fact, the space $B O(2)$ is not simple. 
We can describe it as a Borel construction $\mathbb{C P}^{\infty} \times_{\mathbb{Z} / 2} E(\mathbb{Z} / 2)$, where $\mathbb{Z} / 2$ acts on $B S O(2)=\mathbb{C P}^{\infty}$ by complex conjugation (this changes the sign of the generator of $\left.\pi_{2}\right)$ and freely on $E(\mathbb{Z} / 2)$ as usual.

Next consider a closely related space $B=\left(\mathbb{C P}^{\infty} \times \mathbb{C P}^{\infty}\right) \times_{\mathbb{Z} / 2} E(\mathbb{Z} / 2)$, where $\mathbb{Z} / 2$ acts on both copies of $\mathbb{C P}^{\infty}$ by complex conjugation. Note that there is a fibration $K(\mathbb{Z}, 2) \rightarrow B \stackrel{\psi}{\rightarrow} B O(2)$, where the double cover of $\psi$ is the identity on the second $\mathbb{C P}^{\infty}$ factor and the fiber is the first $\mathbb{C P}^{\infty}$ factor. The universal cover $\widetilde{B}$ of $B$ is homotopy equivalent to $K\left(\mathbb{Z}^{2}, 2\right)$. Since $B$ is obtained via the Borel construction, the cohomology ring of $B$ is $H_{\mathbb{Z} / 2}^{*}\left(\mathbb{C P}^{\infty} \times \mathbb{C P}^{\infty}, \mathbb{Z}\right)$, which can be computed via the spectral sequence with $E_{2}^{p, q}=H^{p}\left(\mathbb{Z} / 2, H^{q}\left(\mathbb{C P}^{\infty} \times \mathbb{C P}^{\infty}, \mathbb{Z}\right)\right)=H^{p}\left(\mathbb{Z} / 2, \mathbb{Z}\left[c_{1}, c_{2}\right]\right)$, where the generators $c_{1}$ and $c_{2}$ are each in degree $q=2$. The generator of $\mathbb{Z} / 2$ changes the signs of $c_{1}$ and $c_{2}$, and is thus given by multiplication by $(-1)^{k}$ on monomials in $c_{1}$ and $c_{2}$ of total degree $q=2 k$. Thus $E_{2}^{0,4}=H^{0}\left(\mathbb{Z} / 2, \mathbb{Z} c_{1}^{2} \oplus\right.$ $\left.\mathbb{Z} c_{1} c_{2} \oplus \mathbb{Z} c_{2}^{2}\right) \cong \mathbb{Z}^{3}$, since the $\mathbb{Z} / 2$ action is trivial. From this one can see that the edge homomorphism $H^{4}(B, \mathbb{Z}) \rightarrow H^{4}\left(\mathbb{C P}^{\infty} \times \mathbb{C P}^{\infty}, \mathbb{Z}\right)$ is split surjective. Thus there is a principal $K(\mathbb{Z}, 3)$ bundle over $B$

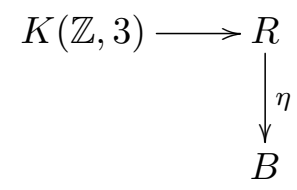

with $k$-invariant corresponding to $c_{1} c_{2} \in H^{4}\left(\mathbb{C P}^{\infty} \times \mathbb{C P}^{\infty}, \mathbb{Z}\right)$, and this defines the space $R$ up to homotopy equivalence.

Via the composition $R \stackrel{\eta}{\rightarrow} B \stackrel{\psi}{\rightarrow} B O(2)$, we have a map $R \rightarrow B O(2)$ and thus a principal $O(2)$ bundle over $R$ and hence a (non-principal) circle bundle $E \stackrel{p}{\rightarrow} R$. Since $\pi_{1}(R) \cong \mathbb{Z} / 2$ and the composite $R \stackrel{\psi \circ \eta}{\longrightarrow} B O(2) \rightarrow B(\mathbb{Z} / 2)$ induces an isomorphism on $\pi_{1}$, the Stiefel-Whitney class of $p$ is the generator of $H^{1}\left(R, \mathbb{F}_{2}\right) \cong \mathbb{F}_{2}$ and in particular is non-trivial. We have a commuting diagram

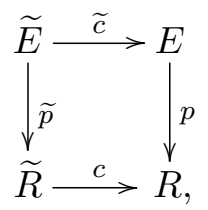

where it is clear that $\widetilde{R}$, the double cover and also the universal cover of $R$, is the classifying space of Bunke and Schick discussed in the statement of the theorem. Here $\widetilde{E}$ is the circle bundle over $\widetilde{R}$ identified by Bunke and Schick, so it has the homotopy type $\widetilde{E} \simeq K(\mathbb{Z}, 2) \times K(\mathbb{Z}, 3)$. Note that 
$\mathbb{Z} / 2 \cong \pi_{1}(E)$ acts trivially on $H^{0}$ and $H^{3}$, but nontrivially on $H^{2}$. Thus one can see that $E \simeq B O(2) \times K(\mathbb{Z}, 3)$. Projection onto the second factor $K(\mathbb{Z}, 3)$ thus defines a canonical class $h \in H^{3}(E, \mathbb{Z})$. So we have a canonical pair $(E \stackrel{p}{\rightarrow} R, h)$ over $R$, and for any space $X$ and map $f: X \rightarrow R$, we get an induced diagram

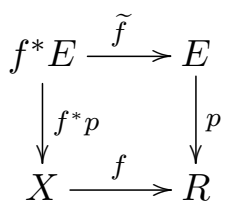

and a pair $\left(f^{*} E, \tilde{f}^{*}(h)\right)$ over $X$, which up to isomorphism only depends on the homotopy class of $f$. We need to prove universality.

First of all, the universal cover $\widetilde{R}$ is what it has to be. For if a space $X$ is simply connected, then any circle bundle over $X$ is automatically principal, since $w_{1}$ must vanish, and a pair in the sense of Definition 2.1 is the same as a pair in the sense of [8, Definition 2.1]. Thus since our $\widetilde{R}$ is the classifying space of Bunke-Schick, we have (again assuming $X$ simply connected)

$$
\mathcal{P}(X)={ }_{\text {Bunke-Schick }}[X, \widetilde{R}] \cong[X, R]
$$

since any map $X \rightarrow R$ has an essentially unique lifting to $\widetilde{R}$. (Really there are two lifts, since a basepoint in the image of $X$ has two inverse images in $\widetilde{R}$, but the two lifts are freely homotopic.)

Now suppose we have a pair $\left(E_{1} \stackrel{p_{1}}{\longrightarrow} X, h_{1}\right)$ over $X$. Then we have the Stiefel-Whitney class $\xi=w_{1}\left(p_{1}\right)$, as well as two classes in $H^{2}\left(X, \mathbb{Z}_{\xi}\right)$ : $c_{1}\left(p_{1}\right)$ and $\left(p_{1}\right)_{!}\left(h_{1}\right)$. These three classes correspond precisely to a homotopy class of maps $g: X \rightarrow B$. (Recall that $B$ is a $\mathbb{Z} / 2$-twisted version of $K(\mathbb{Z}, 2) \times K(\mathbb{Z}, 2)$.) However, the Gysin sequence (Remark 1.2) gives us two additional pieces of information. First of all, the cup product of $c_{1}\left(p_{1}\right)$ and $\left(p_{1}\right)$ ! $\left(h_{1}\right)$ vanishes in $H^{4}(X, \mathbb{Z})$. That says that $g$ factors through the homotopy fiber of a map $k: B \rightarrow K(\mathbb{Z}, 4)$ associated to the product of the two twisted canonical classes. Secondly, the exact sequence says that if $\left(p_{1}\right) !\left(h_{1}\right)$ is known, then $h_{1}$ is determined modulo $\left(p_{1}\right)^{*}\left(H^{3}(X, \mathbb{Z})\right)$. These pieces of information say exactly that $g$ has a lifting to a map $f: X \rightarrow R$, and that the homotopy class of $f$ completely determines the pair $\left(E_{1} \stackrel{p_{1}}{\rightarrow} X, h_{1}\right)$.

Lemma 2.3. The classifying space $R$ of Theorem 2.2 has a unique homotopy involution interchanging the two $K(\mathbb{Z}, 2)$ 's and preserving the homotopy 
class of the map $w_{1}: R \rightarrow K(\mathbb{Z} / 2,1)$ given as the composite

$$
R \stackrel{\eta}{\rightarrow} B \stackrel{\psi}{\rightarrow} B O(2) \stackrel{B q}{\rightarrow} B \mathbb{Z} / 2 .
$$

Proof. This is immediate from the explicit description of $R$; the key point is that the map $k: B \rightarrow K(\mathbb{Z}, 4)$ is symmetric under the interchange of the $K(\mathbb{Z}, 2)$ factors.

Corollary 2.4. Baraglia's Theorem 1.3 holds; that is, every pair has a unique T-dual pair satisfying the axioms of Theorem 1.3.

Proof. The T-dual is gotten by composing the classifying map into $R$ with the involution of Lemma 2.3. Property (1), naturality, and property (2), involutiveness, are immediate. Property (4) follows from the fact that the involution switches the two copies of $K(\mathbb{Z}, 2)$, which from the proof of Theorem 2.2 correspond to $c_{1}(p)$ and $p_{!}(h)$, respectively. Property (3) follows from the fact that the involution of Lemma 2.3 preserves $w_{1}$. It remains just to check the last property, (5). This follows just from checking the universal example $R$. If $E^{\#} \stackrel{p^{\#}}{\longrightarrow} R$ is the circle bundle over $R$ T-dual to the original one (under the involution of Lemma 2.3), then it is easy to see that $E \times{ }_{R} E^{\#}$ is just $K(\mathbb{Z}, 3) \times K(\mathbb{Z} / 2,1)$, and the canonical classes $h \in H^{3}(E, \mathbb{Z})$ and $h^{\#} \in H^{3}\left(E^{\#}, \mathbb{Z}\right)$ both pull back to the canonical class in $H^{3}(K(\mathbb{Z}, 3), \mathbb{Z})$. Since every T-duality is pulled back from the universal one, the theorem follows.

\section{An approach via noncommutative geometry}

Now we aim to reproduce topological T-duality for non-principal circle bundles using the basic idea from [20], which depends on $C^{*}$-algebra crossed products. For this section, we will assume $X$ is a smooth manifold of finite homotopy type and $E \stackrel{p}{\rightarrow} X$ is a circle bundle, not necessarily principal. These conditions are much stronger than they need to $b^{1}$, but they are satisfied in physics applications, and they make the proof of Theorem 3.1 much easier. Let $\widetilde{E} \rightarrow X$ be the principal $O(2)$-bundle associated to $p$. As in Definition 1.1, define the double coverings $c$ of $X$ and $\widetilde{c}$ of $E$ associated to $\xi=w_{1}(p)$. Given $h \in H^{3}(E, \mathbb{Z})$, pull it back to $(\widetilde{c})^{*}(h) \in H^{3}(\widetilde{E}, \mathbb{Z})$, and form the associated stable continuous-trace algebra $A=C T\left(\widetilde{E},(\widetilde{c})^{*}(h)\right)$. We have a free action of $O(2)$ on $\widetilde{E}$, but it may not lift to an action of $O(2)$

\footnotetext{
${ }^{1} X$ need not be a manifold and doesn't really have to have finite homotopy type.
} 
on $A$. We therefore consider the universal cover $G=\widetilde{O}(2)=\mathbb{R} \rtimes H$ of $O(2)$, where $H \cong \mathbb{Z} / 2$ lifts the subgroup $\{1, j\}$ of $O(2)$. Here, as before, $j$ is the reflection matrix $\left(\begin{array}{cc}1 & 0 \\ 0 & -1\end{array}\right)$. Note that $G$ is the affine group of the line. In this context, we have the following.

Theorem 3.1. In this situation of a principal $O(2)$-bundle $\widetilde{E} \rightarrow X$ and a class in $H^{3}(\widetilde{E}, \mathbb{Z})$ pulled back from the non-principal circle bundle $E$ over $X$, the free action of $O(2)$ on $\widetilde{E}$ lifts to an action of $G=\widetilde{O}(2)$ on the stable continuous trace algebra $A=C T\left(\widetilde{E},(\widetilde{c})^{*}(h)\right)$. Furthermore, the action of $G$ on $A$ is uniquely determined up to exterior equivalence once $h \in H^{3}(E, \mathbb{Z})$ is fixed.

The proof uses the obstruction theory developed in [11], which gives necessary and sufficient conditions for such a lifting. The key point is that we can compare the obstructions for $G$ with the similar obstructions for a $\mathbb{Z} / 2$-action lifting the free action of $\mathbb{Z} / 2$ on $\widetilde{E}$ with quotient space $E$. Since the obstruction theory is based on the group cohomology of C. C. Moore [24], further developed by D. Wigner [31], we start with three lemmas about these cohomology groups.

Lemma 3.2. Let $H$ be a finite group and let $A$ be an $H$-module which is a vector space over a field $k$ of characteristic zero with a linear $H$-action. Then $H^{q}(H, A)=0$ for $q>0$.

Proof. This is a standard fact in homological algebra. The point is that the group ring $k H$ is semisimple (by Maschke's Theorem), because of the fact that $k$ has characteristic 0 (in fact it would suffice for the characteristic to be relatively prime to the order of $H$ ), so the functor $A \mapsto A^{H}$ is exact, and has no higher derived functors.

Lemma 3.3. Let $H=\mathbb{R}$ be the real line viewed as a topological group.

(1) Let $A$ be a countable discrete $\mathbb{R}$-module. Then $H^{q}(H, A)=0$ for $q>0$ (and is just $A$ itself for $q=0$ ).

(2) Let $A$ be an $\mathbb{R}$-module which is a complete metrizable topological vector space. Then $H^{q}(H, A)$ (for Moore's cohomology theory) is a real vector space which vanishes for $q>1$.

Proof. For the first fact, see [20, Corollary 4.3], which is based on [31]. For the second, see [20, Corollary 4.7]. 
Lemma 3.4. Let $G=\mathbb{R} \rtimes H, H=\{1, j\} \cong \mathbb{Z} / 2$ acting on $\mathbb{R}$ by $j \cdot t=-t$, $t \in \mathbb{R}$, and let $X$ be a locally compact second countable $G$-space with finite homotopy type. Give $C(X, \mathbb{T})$ the $G$ action coming from the action of $G$ on $X$. Then the maps $H^{q}(G, C(X, \mathbb{T})) \rightarrow H^{q}(H, C(X, \mathbb{T}))$ induced by the inclusion $H \hookrightarrow G$ are isomorphisms for $q=2,3$.

Proof. Since $X$ has finite homotopy type, $M=H^{s}(X, \mathbb{Z})$ is discrete and countable for any $s$, and $\mathbb{R} \triangleleft G$ acts trivially on it. Thus by Lemma 3.3(1), $H^{q}(\mathbb{R}, M)=0$ for $q>0$.

Now we have an exact sequence of topological $G$-modules $[20, \S 4.2$, Equation (4)]

$$
0 \rightarrow H^{0}(X, \mathbb{Z}) \rightarrow C(X, \mathbb{R}) \rightarrow C(X, \mathbb{T}) \rightarrow H^{1}(X, \mathbb{Z}) \rightarrow 0 .
$$

We have just observed that the $\mathbb{R}$-cohomology of the discrete modules $H^{s}(X, \mathbb{Z}), s=0,1$, vanishes past degree 0 . And $H^{q}(\mathbb{R}, C(X, \mathbb{R}))$ is a topological vector space for $q=0,1$, and vanishes for $q>1$, by Lemma 3.3(2). Now use the spectral sequence $H^{p}\left(H, H^{q}(\mathbb{R}, C(X, \mathbb{R})) \Rightarrow H^{p+q}(G, C(X, \mathbb{R}))\right.$. The spectral sequence has $E_{2}^{p, q}=0$ for $q>1$. Since $H^{q}(\mathbb{R}, C(X, \mathbb{R}))$ is a real vector space for $q=0,1$ and $H$ is finite cyclic, we see by Lemma 3.2 that $E_{2}^{p, q}=0$ for $p>0$. Thus (since we've also seen that $E_{2}^{p, q}=0$ for $q>1$ ) $H^{k}(G, C(X, \mathbb{R}))=0$ for all $k>1$. Similarly $H^{k}(H, C(X, \mathbb{R}))=0$ for all $k>$ 0 by Lemma 3.2 .

Now split (1) into two short exact sequences

$$
0 \rightarrow H^{0}(X, \mathbb{Z}) \rightarrow C(X, \mathbb{R}) \rightarrow C(X, \mathbb{T})_{0} \rightarrow 1
$$

and

$$
1 \rightarrow C(X, \mathbb{T})_{0} \rightarrow C(X, \mathbb{T}) \rightarrow H^{1}(X, \mathbb{Z}) \rightarrow 0 .
$$

From (2), we find that the $G$ - or $H$-cohomology of $C(X, \mathbb{T})_{0}$ past degree 1 coincides with the $H$-cohomology of the discrete module $H^{0}(X, \mathbb{Z})$ one degree higher. The conclusion of the lemma now follows by applying the long exact cohomology sequence of (3) along with the spectral sequences $H^{p}\left(H, H^{q}\left(\mathbb{R}, H^{s}(X, \mathbb{Z})\right) \Rightarrow H^{p+q}\left(G, H^{s}(X, \mathbb{Z})\right), s=0,1\right.$.

Proof of Theorem 3.1. We could use the obstruction theory developed in [11], which gives necessary and sufficient conditions for such a lifting. The key point is that we can compare the obstructions for $G$ with the similar obstructions for a $\mathbb{Z} / 2$-action lifting the free action of $\mathbb{Z} / 2$ on $\widetilde{E}$ with quotient 
space $E$. But since the algebra $A$ is the pull-back of the algebra $C T(E, h)$ along the covering map $\widetilde{c}$, such a $\mathbb{Z} / 2$-action exists by [27]. (In fact, by [11, $\S 6.2]$, pull-back induces an isomorphism $\operatorname{Br}_{H}(\widetilde{E}) \cong \operatorname{Br}(E)$ for the equivariant Brauer group.) Thus the obstructions vanish for $\mathbb{Z} / 2$, and so we will see that this implies they also vanish for $G$.

However the simplest argument for existence uses bundle theory and connections. By [28, Lemma 7.1], lifting the $G$-action on $\widetilde{E}$ to a $G$-action on $A$ is equivalent to lifting the $G$-action to the principal $P U$-bundle associated to $A$. Let $P \rightarrow E$ be a principal $P U$-bundle with Dixmier-Douady class $h$; we can assume it is smooth. Choose a connection for $P$ (recall that this gives a canonical way to lift vector fields from $E$ to $P$ ) and take the pull-back connection (under the covering map $\widetilde{c}$ ) on the pull-back bundle $\widetilde{P} \rightarrow \widetilde{E}$. The $\mathbb{R}$-action on $\widetilde{E}$ with quotient $\widetilde{X}$ is defined by a smooth integrable vector field $V$, which lifts via the connection to a horizontal vector field $\widetilde{V}$ on $\widetilde{P}$. Since the connection is pulled back under $\widetilde{c}$ from $P$, it is $H$-invariant. In other words, the pull-back action of $j \in H$ on $\widetilde{P}$ preserves horizontal vectors. Since the action of $j$ conjugates $V$ to $-V$, it must also conjugate $\widetilde{V}$ to $-\widetilde{V}$ (since horizontal lifts of tangent vectors are unique). Thus $\widetilde{V}$ integrates to an $H$-equivariant action of $\mathbb{R}$ on $\widetilde{P}$, that is, to a $G$-action of the type we require.

The alternative argument for existence uses [11, Theorems 4.9 and 5.1], together with Lemma 3.4. We note that the domain and range of the maps $d_{2}, d_{2}^{\prime}$, and $d_{3}$ of those theorems are naturally the same for both $H$ and $G$. A diagram chase then shows that the obstructions are the same for both groups, and since we have a pull-back action of $H$ on $A$, there must also be a $G$-action.

Now we come to the issue of uniqueness. For this we use the exact sequence of [12, Theorem 4], which is also contained in [11, Theorem 5.1]. In fact, we use this exact sequence twice, once for $G$ and once for $H$, plus the result $\operatorname{Br}_{H}(\widetilde{E}) \cong \operatorname{Br}(E)$ from $[11, \S 6.2]$. The exact sequence, together with the fact that we have a forgetful map $\operatorname{Br}_{G}(\widetilde{E}) \rightarrow \operatorname{Br}_{H}(\widetilde{E})$ induced by the inclusion $H \hookrightarrow G$, gives a commuting diagram

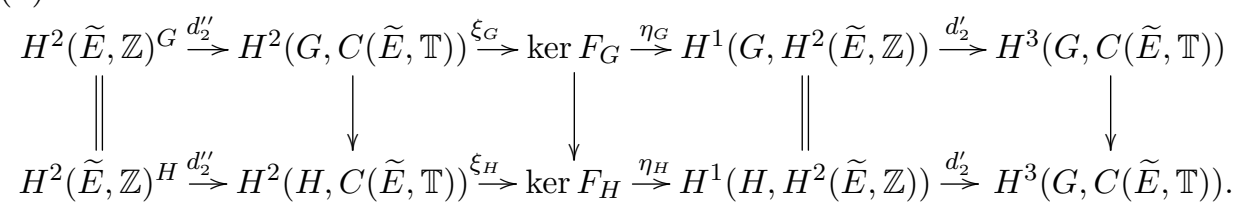


Here $F_{G}$ is the forgetful map $\operatorname{Br}_{G}(\widetilde{E}) \rightarrow \operatorname{Br}(\widetilde{E})$, and similarly $F_{H}$ is the forgetful map $\operatorname{Br}_{H}(\widetilde{E}) \rightarrow \operatorname{Br}(\widetilde{E})$. The vertical equal signs are canonical isomorphisms by Lemma 3.3(1). By Lemma 3.4, the first and last downward arrows in (4) are also isomorphisms. It follows by (4) and the Five-Lemma that the forgetful map $\operatorname{ker} F_{G} \rightarrow \operatorname{ker} F_{H}$ is an isomorphism. But we already know that $\operatorname{Br}_{H}(\widetilde{E}) \cong \operatorname{Br}(E)$ via pull-back under $\widetilde{c}$. So this proves the uniqueness statement.

Theorem 3.5. In the situation of Theorem 3.1, let $A=C T\left(\widetilde{E}, \widetilde{c}^{*}(h)\right)$ and let $\alpha$ be an action of $G=\widetilde{O}(2)$ lifting the free action of $\mathbb{Z} / 2$ on $\widetilde{E}$ with quotient space $E$. Then the crossed product $A \rtimes_{\alpha} G$ is of the form $C T\left(E^{\#}, h^{\#}\right)$ for $p_{\widetilde{E}}^{\#}: E^{\#} \rightarrow X$ some (non-principal) circle bundle over $X$ and $h^{\#} \in$ $H^{3}\left(\widetilde{E}^{\#}, \mathbb{Z}\right)$. Furthermore, $w_{1}\left(p^{\#}\right)=w_{1}(p)$, and the other conditions in Theorem 1.3 are also satisfied.

Proof. Note that $A \rtimes_{\alpha} G \cong\left(A \rtimes_{\alpha} \mathbb{R}\right) \rtimes H$. Since $A$ is the stable continuoustrace algebra associated to an H-flux on a principal $S^{1}$-bundle over $\widetilde{X}$, the theory developed in [20] applies, and $A \rtimes_{\alpha} \mathbb{R}$ is a stable continuoustrace algebra with spectrum the T-dual principal $S^{1}$-bundle $\widetilde{E}^{\#}$ over $\widetilde{X}$ and Dixmier-Douady class the T-dual H-flux (for T-duality of principal bundles over $\widetilde{X}$ ). Since the action of $H$ on $A$ induces the free $\mathbb{Z} / 2$-action on $\widetilde{X}$ with quotient $X$, the action of $H$ on the spectrum $\widetilde{E}^{\#}$ of $A \rtimes_{\alpha} \mathbb{R}$ must also be free. Furthermore, $A \rtimes_{\alpha} G \cong\left(A \rtimes_{\alpha} \mathbb{R}\right) \rtimes H$ will have continuous trace [26, Theorem 1.1], and will be stable since $A$ is stable, and thus will be of the form $C T\left(E^{\#}, h^{\#}\right)$ for some $h^{\#} \in H^{3}\left(E^{\#}, \mathbb{Z}\right)$. By Takai duality (for $H$ ), $A \rtimes_{\alpha} \mathbb{R} \cong C T\left(E^{\#}, h^{\#}\right) \rtimes \widehat{H}$ for the dual action of $\widehat{H} \cong H$. Via [27, Proposition 1.5], we conclude that $A \rtimes_{\alpha} \mathbb{R}$ is the pull-back of $C T\left(E^{\#}, h^{\#}\right)$ along a principal $\widehat{H}$-bundle. So we get a commuting diagram of bundles

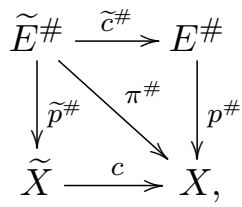

and the spectrum $E^{\#}$ of $A \rtimes_{\alpha} G$ is a non-principal $S^{1}$-bundle over $X$ with the same $w_{1}$ as $E$ (since the associated double cover is again $\widetilde{X} \stackrel{c}{\rightarrow} X$ ). The Dixmier-Douady class of $A \rtimes_{\alpha} \mathbb{R}$ is $\left(\widetilde{c}^{\#}\right)^{*}\left(h^{\#}\right)$.

Now we can check the condition of Theorems 1.3. Conditions (1) and (3) are now obvious. Condition (2), that T-duality is involutive, follows from 
Takai duality, since when we T-dualize a second time, we get

$$
\left(\left(\widetilde{c}^{\#}\right)^{*}\left(A \rtimes_{\alpha} G\right)\right) \rtimes_{\alpha^{\#}} G \cong\left(\left(A \rtimes_{\alpha} \mathbb{R}\right) \rtimes_{\widehat{\alpha}} \mathbb{R}\right) \rtimes H \cong A \rtimes H \cong C T(E, h) .
$$

Condition (4) follows from the corresponding condition for T-duality of principal circles bundles over $\widetilde{X}$, along with the relationships between $c_{1}(\widetilde{p})$ and $c_{1}(p)$, etc.

To check condition (5), note that we get from [26, Proposition 2.1 and Theorem 2.2] a commuting diagram of principal circle bundles

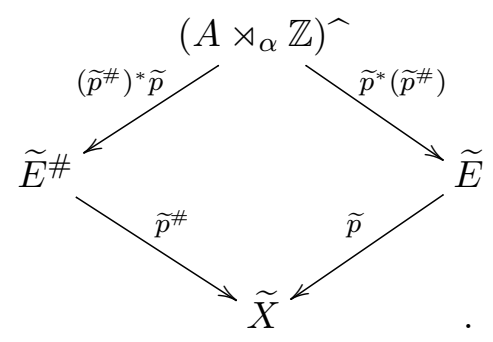

Furthermore $A \rtimes_{\alpha} \mathbb{Z} \cong\left(A \rtimes_{\alpha} \mathbb{R}\right) \rtimes_{\widehat{\alpha}} \mathbb{Z}$ has Dixmier-Douady class equal to the pull-backs of both $\widetilde{c}^{*}(h)$ and $\left(\widetilde{c}^{\#}\right)^{*}\left(h^{\#}\right)$. This diagram fits into a larger diagram of circle bundles and covering maps

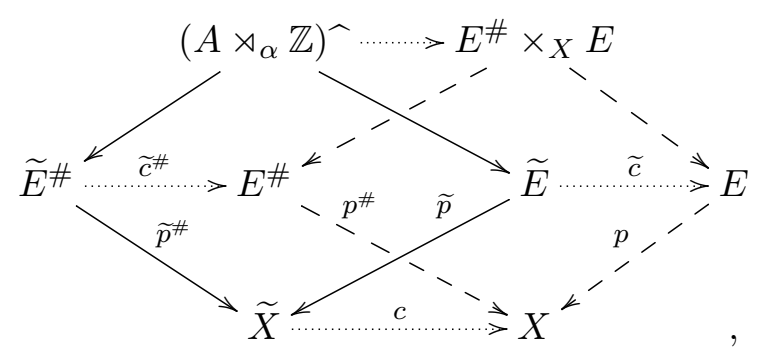

where for simplicity we've left off the labels on the maps in the top half of the diagram and we have used dotted arrows for 2-fold covering maps and dashed arrows for non-principal circle bundles. Now over the space $E^{\#} \times_{X} E$ we have the continuous-trace algebra $\left(A \rtimes_{\alpha} \mathbb{Z}\right) \rtimes H$. The algebra $A \rtimes_{\alpha} \mathbb{Z}$ is pulled back from $A$ by [27, Theorem 2.5], and $A$ is pulled back from $C T(E, h)$, so by commutativity of (6), $A \rtimes_{\alpha} \mathbb{Z}$ is pulled back from the algebra $\left(A \rtimes_{\alpha} \mathbb{Z}\right) \rtimes H$ over $E^{\#} \times_{X} E$, which in turn is pulled back from $C T(E, h)$. Thus the Dixmier-Douady invariant of $A \rtimes_{\alpha} \mathbb{Z}$ is the pull-back of $h \in H^{3}(E, \mathbb{Z})$. A similar argument using the dual action shows that it is also equal to the pull-back of $h^{\#} \in H^{3}\left(E^{\#}, \mathbb{Z}\right)$. So this proves property (5) of Theorem 1.3 and concludes the proof. 


\section{4. $K$-theory of certain crossed products}

Definition 4.1. We begin with a fact about equivariant $K$-theory for a compact group $H$, namely that it is $R O(H)$-graded (see for example [23, Chapters IX, X, XIII, and XIV]). Given a compact group $H$, an $H-C^{*}-$ algebra $A$, and an orthogonal representation of $H$ on a finite-dimensional real vector space $V$, we can twist $H$-equivariant $K$-theory of $A$ by $V$, getting $K_{i}^{H, V}(A)=K_{i}\left(A \otimes C_{0}(V)\right)$, where $H$ acts on the second factor via the linear representation and acts on the tensor product by the tensor product action. Note that if $V$ happens to be a complex vector space and the action of $H$ is complex linear, then equivariant Bott periodicity gives an isomorphism $K_{*}^{H, V} \cong K_{*}^{H}$. (This is also true more generally if $V$ is even-dimensional over $\mathbb{R}$ and if the action of $H$ factors through $\operatorname{Spin}^{c}(V)$.) And if $H$ acts trivially on $V, K_{*}^{H, V} \cong K_{*+\operatorname{dim} V}^{H}$. But in general, the groups $K_{*}^{H, V}$ are not the same as $K_{*}^{H}$, even modulo a grading shift. In the noncommutative world, another approach to the groups $K_{*}^{H, V}$ is possible via graded Clifford algebras, since $C_{0}(V)$ is $K K^{H}$-equivalent to $\mathrm{C} \ell(V)$, the complex Clifford algebra of $V$ viewed as a graded $H$-algebra [5, Theorem 20.3.2]. But this requires introducing graded $C^{*}$-algebras, which we'd prefer to avoid.

For our purposes we will need only the group $H=\mathbb{Z} / 2$, which has two real characters, the trivial character 1 and the non-trivial character $t$, the sign representation - (where the generator of the group acts by -1 on $\mathbb{R}$ ). Thus we have twisted equivariant $K$-groups $K_{*}^{\mathbb{Z} / 2,-}$, which are discussed in greater detail in [29]. These are modules over the representation ring $R=$ $R(H) \cong \mathbb{Z}[t] /\left(t^{2}-1\right)$, which has two special complementary prime ideals, $I=(t-1)$ and $J=(t+1)$. The coefficient groups for $K_{*}^{\mathbb{Z} / 2,-}$ are computed in [16], for example. It turns out that $K_{*}^{\mathbb{Z} / 2,-}(\mathbb{C}) \cong \mathbb{Z}$ (actually $R / J$ as an $R$-module) for $* \equiv 0(\bmod 2)$ and $\cong 0$ for $* \equiv 1(\bmod 2)$. Twisting twice brings us back to conventional equivariant $K$-theory since a direct sum of two copies of the sign character is a complex representation, where equivariant Bott periodicity applies.

Remark 4.2. Let $H=\mathbb{Z} / 2, R=R(H)$, and let $A$ be an $H$-algebra. It is important to note that the equivariant $K$-groups $K_{*}^{H}(A)$ (even as $R$ modules) do not determine the groups $K_{*}^{H,-}(A)$ in general. This is due to the failure of the equivariant Künneth Theorem for $K_{*}^{H}$ localized at prime ideals $P$ containing the augmentation ideal $I$. (See $[25$, p. 235].) Here is a way to construct a very specific counterexample. It is known that the Cuntz $C^{*}$-algebra $\mathcal{O}_{2}$ has vanishing $K$-groups and is very "nice" (nuclear and in the "bootstrap class" where the universal coefficient theorem holds for $K K$ ). 
Furthermore, it is known (originally due to Blackadar (unpublished), but see [18, Lemma 4.7] for a specific construction) that there are actions of $H$ on $\mathcal{O}_{2}$ for which $\mathcal{O}_{2} \rtimes H$ has nontrivial, but uniquely 2-divisible, $K$-groups, say $K_{0}\left(\mathcal{O}_{2} \rtimes H\right) \cong \mathbb{Z}\left[\frac{1}{2}\right]$. Choose such an action and let $A=\mathcal{O}_{2} \rtimes H$, which is an $H$-algebra under the dual action to the original action on $\mathcal{O}_{2}$. (Here we are identifying $H$ with its dual in the obvious way.) By Takai Duality and the Green-Julg Theorem, $K_{*}^{H}(A) \cong K_{*}(A \rtimes H) \cong K_{*}\left(\mathcal{O}_{2}\right)=0$, while $K_{*}(A) \neq$ 0 . Incidentally, one could also construct an abelian $H$ - $C^{*}$-algebra $A$ with $K_{*}(A)=0$ but $K_{*}^{H}(A) \neq 0$ by using deep results from algebraic topology, and then proceed similarly with $A$ in place of $\mathcal{O}_{2}$; see [28, Lemma 5.7] and $[29]$.

Proposition 4.3. With $H=\mathbb{Z} / 2$ and $A$ the $H-C^{*}$-algebra defined in Remark 4.2, $K_{*}^{H}(A)=0$ but $K_{*}^{H,-}(A) \neq 0$.

Proof. We have $K_{*}^{H,-}(A)=K_{*}\left(A \otimes C_{0}\left(\mathbb{R}^{-}\right)\right)$, Since $\mathbb{R}^{-}$equivariantly contains $\{0\}$ (a fixed point) and $\mathbb{R}^{-} \backslash\{0\}$ is equivariantly homeomorphic to $H \times \mathbb{R}$ (with the $H$-action interchanging the two connected components), we get a short exact sequence of $H$-algebras

$$
0 \rightarrow A \otimes C(H) \otimes C_{0}(\mathbb{R}) \rightarrow A \otimes C_{0}\left(\mathbb{R}^{-}\right) \rightarrow A \rightarrow 0
$$

and thus a long exact sequence of equivariant $K$-groups

$$
\cdots \rightarrow K_{*+1}^{H}(A) \rightarrow K_{*+1}(A) \rightarrow K_{*}^{H,-}(A) \rightarrow K_{*}^{H}(A) \rightarrow \cdots .
$$

This exact sequence appears as [29, Proposition 3.3]. Since $K_{*}^{H}(A)=0$ (in all degrees), we obtain $K_{*}^{H,-}(A) \cong K_{*+1}(A) \neq 0$.

Corollary 4.4 (of the proof). Let $H=\mathbb{Z} / 2$ and let $A$ be an $H-C^{*}$ algebra. Then there is an exact sequence of $K$-groups

$$
\cdots \rightarrow K_{*+1}^{H}(A) \rightarrow K_{*+1}(A) \rightarrow K_{*}^{H,-}(A) \rightarrow K_{*}^{H}(A) \rightarrow \cdots .
$$

In particular, if both $K_{*}^{H}(A)=0$ and $K_{*}(A)=0$, then $K_{*}^{H,-}(A)=0$.

In order to use the theory developed in Section 3, we need to be able to compute the $K$-theory of crossed products by $\widetilde{O}(2)$. The following result, modeled on Connes's "Thom Isomorphism Theorem" of [10], is precisely what is needed. 
Theorem 4.5. Let $A$ be a $C^{*}$-algebra equipped with an action of $G=\widetilde{O}(2)$. Note that by restriction to the copy of $H=\mathbb{Z} / 2$ in $G$ gotten by lifting $\{1, j\} \subset$ $O(2), A$ is also a $\mathbb{Z} / 2$-algebra. Then the $K$-theory of $A \rtimes G$ is naturally isomorphic to $K_{*}^{\mathbb{Z} / 2,-}(A)$ as defined in Definition 4.1 .

Proof. Note that $A$ being a $G$-algebra means that $A$ is both a $\mathbb{Z} / 2$-algebra and an $\mathbb{R}$-algebra, and that the actions $\alpha$ of $\mathbb{R}$ and $\gamma$ of $\mathbb{Z} / 2$ are related by the commutation relation $\gamma_{j}\left(\alpha_{t}(a)\right)=\alpha_{-t}\left(\gamma_{j}(a)\right)$. (Here $j$ denotes the nontrivial element of $\mathbb{Z} / 2$.) This commutation rule comes from the multiplication rule in $G$. Note also for future use that the action $\gamma$ of $j$ on $\mathbb{R}^{-}$(the superscript is to emphasize that the action is by reflection) also induces an action of $j$ on the Pontrjagin dual group $\widehat{\mathbb{R}} \cong \mathbb{R}^{*}$ (the dual vector space), again given by reflection. By the isomorphism $A \rtimes G \cong\left(A \rtimes_{\alpha} \mathbb{R}\right) \rtimes_{\gamma} \mathbb{Z} / 2$ and the GreenJulg Theorem [19], $K_{*}(A \rtimes G)$ is naturally isomorphic to $K_{*}^{\mathbb{Z} / 2}\left(A \rtimes_{\alpha} \mathbb{R}\right)$. We want to show that this in turn is isomorphic to $K_{*}^{\mathbb{Z} / 2}\left(A \otimes C_{0}\left(\mathbb{R}^{-}\right)\right)$, the equivariant $K$-theory of the crossed product for the trivial action of $\mathbb{R}$ on $A$. Here the $\mathbb{R}$ is really the dual group $\widehat{\mathbb{R}}$ of the original copy of $\mathbb{R}$, but since $\mathbb{Z} / 2$ acts on $\widehat{\mathbb{R}}$ here by the sign representation, the result then follows.

First we construct natural homomorphisms

$$
\phi_{\alpha}^{*}: K_{*}^{H}\left(A \otimes C_{0}\left(\mathbb{R}^{-}\right)\right) \rightarrow K_{*}^{H}\left(A \rtimes_{\alpha} \mathbb{R}\right)
$$

as follows. Let $C A=C_{0}([0,1), A) \cong C_{0}([0,1)) \otimes A$ be the cone on $A$. Define an action of $G$ on $C A$ by letting $H$ act by its original action on $A$ and act trivially on $[0,1)$. The real line $\mathbb{R}$ is defined to act on $C A$ by $\beta_{t}(f)(s)=\alpha_{s t}(f(s))$. This is compatible with the action of $H$ because of the commutation relation $\gamma_{j}\left(\alpha_{t}(a)\right)=\alpha_{-t}\left(\gamma_{j}(a)\right)$, so we have an action of $G$ whose restriction to $\mathbb{R}$ is $\beta$. Since the action of $G$ commutes with the action of $C^{b}([0,1))$ by central multipliers, we have a $G$-equivariant exact sequence

$$
0 \rightarrow C_{0}((0,1), A) \rightarrow C A \stackrel{\text { eval at } 0}{\longrightarrow} A \rightarrow 0
$$

and thus a short exact sequence of $H$-algebras

$$
0 \rightarrow C_{0}((0,1), A) \rtimes_{\beta} \mathbb{R} \rightarrow C A \rtimes_{\beta} \mathbb{R} \stackrel{\text { eval at } 0}{\longrightarrow} A \rtimes \mathbb{R} \rightarrow 0 .
$$

But the action of $\mathbb{R}$ on the copy of $A$ over $0 \in[0,1)$ is trivial, so the crossed product on the right is $A \otimes C_{0}\left(\mathbb{R}^{-}\right)$(as an $H$-algebra). Over each point of $(0,1)$, the action of $\mathbb{R}$ on the associated copy of $A$ is just a rescaling of $\alpha$, and in fact we claim that $C_{0}((0,1), A) \rtimes_{\beta} \mathbb{R} \cong C_{0}((0,1)) \otimes\left(A \rtimes_{\alpha} \mathbb{R}\right)$ 
( $H$-equivariantly). Granting this, the boundary map in the long exact $K^{H_{-}}$ theory sequence attached to $(7)$ gives the desired natural map $\Phi$.

To prove the claim, observe that both of the algebras $C_{0}((0,1), A) \rtimes_{\beta} \mathbb{R}$ and $C_{0}((0,1)) \otimes\left(A \rtimes_{\alpha} \mathbb{R}\right)$ are completions of the algebra $C_{c}((0,1) \times \mathbb{R}, A)$, but for different convolution products. If $s$ denotes the coordinate on $(0,1)$ and $t$ the coordinate on $\mathbb{R}$, the product is

$$
\left(f \star_{1} g\right)(s, t)=\int f(s, u) \alpha_{s u} g(s, t-u) d u
$$

in the first case and

$$
\left(f \star_{2} g\right)(s, t)=\int f(s, u) \alpha_{u} g(s, t-u) d u
$$

in the second case. These products are intertwined by the map $f \mapsto f^{\prime}$, where $f^{\prime}(s, t)=s^{-1} f\left(s, s^{-1} t\right)$, which is clearly $H$-equivariant, and one can easily see this extends to the desired isomorphism of crossed products.

Next, it is clear that the maps $\phi_{\alpha}^{*}$ satisfy the following (taken almost verbatim from $[10, \S I I])$ :

\section{Axioms 4.6 (following Connes).}

(1) If $A=\operatorname{End}(V)$ for some finite-dimensional complex representation $V$ of $H=\mathbb{Z} / 2$, equipped with the trivial action of $\mathbb{R}$, then $\phi_{\alpha}^{*}$ is the canonical isomorphism.

(2) (Naturality) $\phi$ is natural for maps of G-algebras.

(3) (Compatibility with suspension) If $S \alpha$ is the suspension of $\alpha$, acting on $S A=A \otimes C_{0}(\mathbb{R})$ (with trivial $G$-action on the second factor), and $s_{A}^{j}: K_{j}(A) \rightarrow K_{j+1}(S A)$ is the usual isomorphism, then $\phi_{S \alpha}^{j+1} \circ s_{A}^{j}=$ $s_{A \rtimes_{\alpha} \mathbb{R}}^{j} \circ \phi_{\alpha}^{j}$.

We follow the outline of the proof in [10], starting with the following lemma.

Lemma 4.7 (Cf. [10, II, Proposition 4]). In the situation of Theorem 4.5, suppose $p$ is an $H$-fixed self-adjoint projection in $A$ and $t \mapsto \alpha_{t}(p)$ is smooth. Then there is an exterior equivalent action of $G$ on $A$ fixing $p$.

Proof. Let $\delta$ be the unbounded derivation which is the infinitesimal generator of the action of $\mathbb{R}$. Then $\delta$ maps $A^{\infty}$, the smooth vectors for the 
action of $G$, into itself, $\delta$ commutes with $*$, and $\alpha_{t}(a)=e^{t \delta(a)}$. In the terminology of [10], $\delta(a)=i[M, a]$, where $M$ is a self-adjoint "unbounded multiplier" of $A$ (and a genuine multiplier of $A^{\infty}$ ), so $\alpha_{t}(a)=e^{i t \operatorname{ad} M}(a)=$ $\operatorname{Ad}\left(e^{i t M}\right)(a)=e^{i t M} a e^{-i t M}$. (We've changed Connes' $H$ to an $M$ to avoid confusion with the group $H=\mathbb{Z} / 2$.) Since $p$ is $H$-fixed, $j \cdot p=p$. On the other hand, $j \cdot M=-M$ because of the structure of $G$. We simply follow the same method as in [10]. Let $M^{\prime}=p M p+(1-p) M(1-p)$. This is again an unbounded multiplier of $A$ (and a genuine multiplier of $A^{\infty}$ ), and it clearly commutes with $p$. Furthermore, $M^{\prime}$ is a bounded perturbation of $M$, and so defines an exterior-equivalent action of $\mathbb{R}$ fixing $p$ (as shown in [10]). This action extends to an action of $G$ since $j \cdot M^{\prime}=-M^{\prime}$ (since $j \cdot p=p$ and $j \cdot M=-M)$.

Proof of Theorem 4.5 (cont'd). Now we return to Connes' strategy for proving Theorem 4.5. Propositions 1, 2, and 3 in [10, II] go through without change and show that we may assume $A$ is unital, and that we are free to replace $A$ by a matrix algebra over $A$ whenever necessary. Another simple observation is that replacing $A$ by $A \otimes C_{0}\left(\mathbb{R}^{-}\right)$(with $\mathbb{R}$ acting trivially on the second factor) gives us maps

$$
\begin{aligned}
\phi_{\alpha \otimes 1}^{*}: & K_{*}^{H}\left(A \otimes C_{0}\left(\mathbb{R}^{-}\right) \otimes C_{0}\left(\mathbb{R}^{-}\right)\right) \rightarrow K_{*}^{H}\left(\left(A \otimes C_{0}\left(\mathbb{R}^{-}\right)\right) \rtimes_{\alpha \otimes 1} \mathbb{R}\right) \\
\text { or } \quad \phi_{\alpha \otimes 1}^{*}: & K_{*}^{H}(A) \rightarrow K_{*}^{H,-}\left(A \rtimes_{\alpha} \mathbb{R}\right),
\end{aligned}
$$

where we've used equivariant Bott periodicity once. Furthermore, $\phi_{\alpha}^{*}$ is an isomorphism for all $A$ if and only if $\phi_{\alpha \otimes 1}^{*}$ is an isomorphism for all $A$ (since we can tensor with $C_{0}\left(\mathbb{R}^{-}\right)$to go back and forth from one to the other).

To finish the proof, we apply Takai Duality, which gives an $H$-equivariant isomorphism $\left(A \rtimes_{\alpha} \mathbb{R}\right) \rtimes_{\widehat{\alpha}} \widehat{\mathbb{R}} \cong A \otimes \mathcal{K}, \mathcal{K}$ the algebra of compact operators on $L^{2}(\mathbb{R})$. Thus putting together $\phi^{*}$ for $A$ and for $A \rtimes_{\alpha} \mathbb{R}$, we get maps

$$
K_{*}^{H}(A) \stackrel{\phi_{\alpha \otimes 1}^{*}}{\longrightarrow} K_{*}^{H,-}\left(A \rtimes_{\alpha} \mathbb{R}\right) \stackrel{\phi_{\alpha}^{*}}{\longrightarrow} K_{*}^{H}(A),
$$

and we just need to show this composite is the identity. Because of compatibility with suspensions (property 4.6(3)), it is enough to do this for $K_{0}^{H}$. Without loss of generality, we may assume $A$ is unital and just consider a class in $K_{0}^{H}(A)$ represented by a finitely generated projective $A$ module with compatible $H$-action. By [5, §11.3], such a module can be represented by an equivariant Murray-von Neumann equivalence class of $H$-invariant projections $p \in \operatorname{End}(V) \otimes A$, for some finite-dimensional $H$-module $V$. Without loss of generality, we may replace $A$ by $\operatorname{End}(V) \otimes A$, with trivial $\mathbb{R}$ action 
on the first factor. Apply Lemma 4.7. This enables us to change the $\mathbb{R}$ action within the same exterior equivalence class so that the action fixes $p$. After these reductions, $p$ defines a $G$-equivariant map $\mathbb{C} \stackrel{p}{\rightarrow} A$. Because of Axioms 4.6 (especially naturality), this reduces us to checking the theorem for $\mathbb{C}$ with trivial action, for which the result is obvious by Axiom 4.6(1).

Now we apply this result to the situation of Section 3.

Corollary 4.8 (cf. [2, Proposition 6.1]). Let $X$ be a smooth manifold of finite homotopy type, and let $p: E \rightarrow X$ be a non-principal circle bundle over $X$ with associated principal $O(2)$-bundle $\widetilde{E} \rightarrow X$. Choose $h \in H^{3}(E, \mathbb{Z})$ and let $A=C T\left(\widetilde{E},(\widetilde{c})^{*}(h)\right)$ with the $G$-action $\alpha$ lifting the free action of $O(2)$ on $\widetilde{E}$ as in Theorem 3.1. Then there is a natural isomorphism $K^{*}(E, h) \cong K^{*+1}(A \rtimes G)$. In other words, in the notation of Theorem 3.5, $K^{*+1}\left(E^{\#}, h^{\#}\right) \cong K^{*}(E, h)$.

Proof. By Theorem 4.5, we may replace $K^{*+1}(A \rtimes G)$ by $K_{H,-}^{*+1}\left(\widetilde{E},(\widetilde{c})^{*}(h)\right)$. Thus we compute

$$
\begin{aligned}
K_{H,-}^{*+1}\left(\widetilde{E}, \widetilde{c}^{*} h\right) & =K_{H}^{*+1}\left(\widetilde{E} \times \mathbb{R}^{-}, \widetilde{c}^{*} h\right) \\
& (\text { since the } H \text {-action is free and is a pull-back action }) \\
& \cong K^{*+1}(E \times \mathbb{R}, h) \cong K^{*}(E, h)
\end{aligned}
$$

\section{Circle fibrations}

It turns out to be necessary for some purposes to consider compactifications of spacetime that are not necessarily circle bundles, but which also have singular fibers. This happens for instance in the the Strominger-Yau-Zaslow (SYZ) formulation [30] of mirror symmetry. In the paper [22], the authors considered a simple case when singular fibers exist, namely in the case of a smooth action of a circle on spacetime which is not necessarily free. In this section, we consider a more general case of a smooth action, which is generically free but not necessarily free, of the affine circle group $O(2)$ on a branched double cover $\widetilde{E}$ of spacetime $E$, with the reflection $j \in O(2)$ acting so that $E=\widetilde{E} /\{1, j\}$. The $O(2)$-action gives rise to the (non-principal) circle "fibration" $E=\widetilde{E} /\{1, j\} \rightarrow X=\widetilde{E} / O(2)$, which in general has singular fibers. We will extend the T-duality picture and isomorphism of the previous sections to include this case. It will be a generalization, both of Baraglia's Theorem 1.3 and the main result in [22]. 
Consider the diagonal action of $O(2)$ on $\widetilde{E} \times E O(\underset{\widetilde{E}}{2})$, with quotient the Borel construction $\widetilde{E}_{O(2)}=\widetilde{E} \times_{O(2)} E O(2)$. Then $p: \widetilde{E}_{\mathbb{Z} / 2}=\widetilde{E} \times_{\mathbb{Z} / 2} E O(2)$ $\rightarrow \widetilde{E}_{O(2)}$ is an honest (non-principal) $S^{1}$-bundle as discussed in Section $1^{2}$, and is classified by the pair of invariants, $\xi=w_{1}(p) \in H^{1}\left(\widetilde{E}_{O(2)}, \mathbb{Z} / 2\right)=$ $H_{O(2)}^{1}(\widetilde{E}, \mathbb{Z} / 2)$ and $c_{1}(p) \in H^{2}\left(\widetilde{E}_{O(2)}, \mathbb{Z}_{\xi}\right)=H_{O(2)}^{2}\left(\widetilde{E}, \mathbb{Z}_{\xi}\right)$. We also assume that $E$ comes with an $H$-flux $h$, which we pull back to an integral 3cohomology class on $\widetilde{E} \times_{\mathbb{Z} / 2} E O(2) \simeq \widetilde{E} \times_{\mathbb{Z} / 2} E \mathbb{Z} / 2=\widetilde{E}_{\mathbb{Z} / 2}$. Then by the Gysin sequence (Remark 1.2 in Section 1), $p_{!}(h) \in H^{2}\left(\widetilde{E}_{O(2)}, \mathbb{Z}_{\xi}\right)$ $=H_{O(2)}^{2}\left(\widetilde{E}, \mathbb{Z}_{\xi}\right)$.

In analogy with the equivariant classification of principal circle bundles [17, Theorem C.47], we can deduce the following.

Theorem 5.1 (O(2)-equivariant circle bundles). Let $M$ be a connected manifold equipped with a smooth action $\alpha$ of $O(2)$. Define an $O(2)$ equivariant principal circle bundle over $M$ to mean a principal circle bundle $\pi: Y \rightarrow M$, defined by a free (smooth) action $\alpha_{2}$ of $S O(2)$ on $Y$ with quotient space $M$, together with an action $\alpha_{1}$ of $O(2)$ on $Y$, so that the restriction of $\alpha_{1}$ to $S O(2)$ commutes with the action $\alpha_{2}$ defined by the bundle structure, $\alpha_{1}$ lifts the original action $\alpha$ of $O(2)$ on $M$, and the action under $\alpha_{1}$ of $j$ (as before, the standard reflection matrix in $O(2)$ ) satisfies $\alpha_{1}(j) \alpha_{2}(t) \alpha_{1}(j)=\alpha_{2}(\bar{t})$ (note the complex conjugation!). This last condition means that $\alpha_{1}(j)$ and $\alpha_{2}$ combine to give another $O(2)$-action (which we again call $\alpha_{2}$ ) on $Y$. Then the pair of invariants, the equivariant first StiefelWhitney class $\xi \in H_{O(2)}^{1}(M, \mathbb{Z} / 2)$ defined by $\alpha$ as above and the equivariant first Chern class $c_{1}^{O(2)} \in H_{O(2)}^{2}\left(M, \mathbb{Z}_{\xi}\right)$, classify $O(2)$-equivariant principal circle bundles $\left(\pi: Y \rightarrow M, \alpha_{1}\right)$ over $M$ up to equivalence.

Proof. By [17, Theorem C.47], the $S O(2)$-equivariant principal circle bundles over $M$ (what one gets by forgetting the action of $j$ everywhere) are classified by $c_{1}^{S O(2)} \in H_{S O(2)}^{2}(M, \mathbb{Z})$. The problem is to augment this result to take the action of $j$ into account. First suppose that an $O(2)$-equivariant principal circle bundle $\left(\pi: Y \rightarrow M, \alpha_{1}\right)$ over $M$ is given. We have already defined $\xi$ as the cohomology class in $H_{O(2)}^{1}(M, \mathbb{Z} / 2)=H^{1}\left(M_{O(2)}, \mathbb{Z} / 2\right)$ defining the double covering $M_{S O(2)} \rightarrow M_{O(2)}$. Just thinking of $\pi: Y \rightarrow M$ as an $S O(2)$-equivariant circle bundle, we have the class $c_{1}^{S O(2)} \in H_{S O(2)}^{2}(M, \mathbb{Z})=$

\footnotetext{
${ }^{2}$ Strictly speaking, we've given up local compactness and homotopy finiteness in doing this, but this is not a serious issue. For most purposes one can replace $E O(2)$ by a finite-dimensional approximation which is actually a manifold.
} 
$H^{2}\left(M_{S O(2)}, \mathbb{Z}\right)$. Since $M_{S O(2)}$ is the double cover of $M_{O(2)}$ defined by $\xi$ and the action $\alpha_{1}$ of $j$ does not commute with the bundle action $\alpha_{2}$ of $S O(2)$,but rather anticommutes, we can also think of this class as living in $H^{2}\left(M_{O(2)}, \mathbb{Z}_{\xi}\right)=H_{O(2)}^{2}\left(M, \mathbb{Z}_{\xi}\right)$.

Now let's go the other way. Assume that $\xi$ (already defined by $\alpha$ ) and $c_{1}^{O(2)} \in H_{O(2)}^{2}\left(M, \mathbb{Z}_{\xi}\right)$ are given. Then we have the principal circle bundle $p: M \times E O(2) \simeq M \rightarrow M_{S O(2)}$ defined by $\alpha$ as well as another principal circle bundle $\pi_{1}: Y_{1} \rightarrow M_{S O(2)}$ defined by $c_{1}^{O(2)}$, or if you prefer, a principal $O(2)$-bundle $\pi_{1}^{\prime}: Y_{1} \rightarrow M_{O(2)}$ with invariants $\left(\xi, c_{1}^{O(2)}\right)$. Forming the fiber product, we get a commuting diagram of principal circle bundles

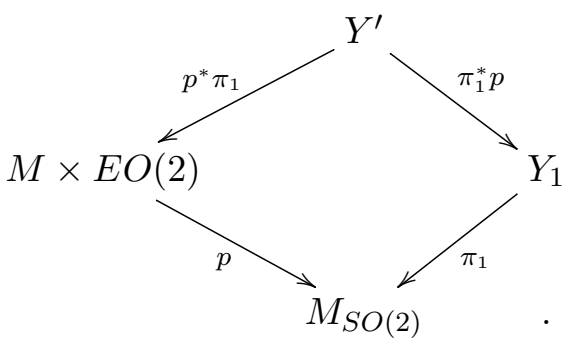

By [17, Theorem C.47], we also have an $S O(2)$-equivariant principal circle bundle $\pi: Y \rightarrow M$ defined by $c_{1}^{O(2)}$, and we can complete the previous diagram to a commutative diagram

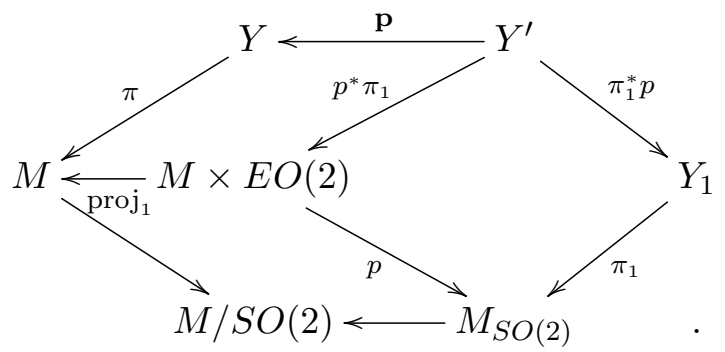

Here the top horizontal arrow $\mathbf{p}$ (pointing to the left) is a map of principal circle bundles, and is equivariant for the $S O(2)$-actions. Since $\pi_{1}^{\prime}: Y_{1} \rightarrow M_{O(2)}$ is a principal $O(2)$-bundle, we also have an action of $j$ on $Y^{\prime}$ with the right intertwining properties, and we just need to show that it descends to $Y$. We want to define $j \cdot y=\mathbf{p}\left(j \cdot y^{\prime}\right)$ when $\mathbf{p}\left(y^{\prime}\right)=y$, so one just needs to check that this is well-defined, i.e., doesn't depend on the choice of $y^{\prime} \in \mathbf{p}^{-1}(y)$. But if also $\mathbf{p}\left(y_{1}^{\prime}\right)=y$, then $y_{1}^{\prime}=t \cdot y^{\prime}$ for some $t \in S O(2)$, and 
then

$$
\mathbf{p}\left(j \cdot y_{1}^{\prime}\right)=\mathbf{p}\left(j \cdot t \cdot y^{\prime}\right)=\mathbf{p}\left(\bar{t} \cdot j \cdot y^{\prime}\right)=\mathbf{p}\left(j \cdot y^{\prime}\right)
$$

as required.

Using this result, we see that the pair of invariants, $\xi \in H_{O(2)}^{1}(\widetilde{E}, \mathbb{Z} / 2)$ and $p_{!}(h) \in H_{O(2)}^{2}\left(\widetilde{E}, \mathbb{Z}_{\xi}\right)$ determine an $O(2)$-equivariant principal circle bundle $\widetilde{Y} \stackrel{\widetilde{\pi}}{\rightarrow} \widetilde{E}$. Since the actions of $O(2)$ and $S^{1}$ on this space commute, we can divide out by the action of $\{1, j\} \cong \mathbb{Z} / 2 \subset O(2)$, getting a principal $S^{1}$ bundle $Y \stackrel{\pi}{\rightarrow} E$. Consider the commutative diagram (the analogue of (5))

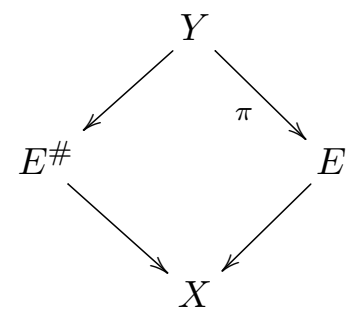

involving the singular spaces $X=\widetilde{E} / O(2)$ and $E^{\#}=\widetilde{Y} / O(2)$. We can replace the singular spaces by their Borel constructions by lifting the above diagram to the following commutative diagram of actual circle bundles:

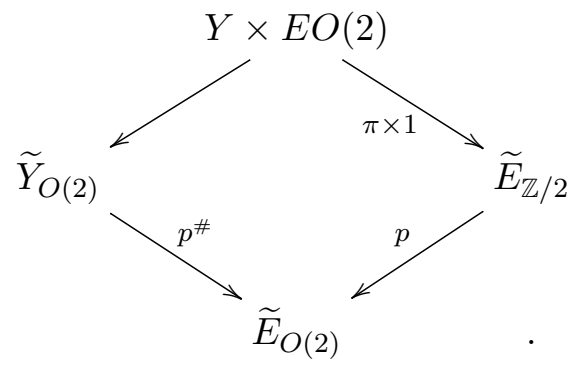

Now we can apply Baraglia's theorem Thm. 1.3 to deduce the existence of the T-dual $H$-flux $\widehat{h} \in H^{3}\left(Y_{O(2)}, \mathbb{Z}\right)=H_{O(2)}^{3}(Y, \mathbb{Z})$ satisfying the properties listed in the theorem. Summarizing all that we've done, we get the following:

Theorem 5.2. Let $\widetilde{E}$ be a connected manifold with an action of $O(2)$ as above, with invariants $\xi=w_{1}(p) \in H_{O(2)}^{1}\left(\widetilde{E}, \mathbb{F}_{2}\right)$ and $c_{1}(p) \in H_{O(2)}^{2}\left(\widetilde{E}, \mathbb{Z}_{\xi}\right)$. Then for any choice of $H$-flux $h \in H_{\mathbb{Z} / 2}^{3}(\widetilde{E}, \mathbb{Z})$, there is a unique $O(2)$ equivariant circle bundle $\widetilde{Y} \stackrel{\widetilde{\pi}}{\rightarrow} \widetilde{E}$ and a unique $T$-dual $H$-flux $h^{\#} \in H_{O(2)}^{3}(\tilde{Y}$, $\mathbb{Z})$, characterized by the following axioms: 
(1) T-duality is natural, so the T-dual of a pull-back is the pull-back of the dual.

(2) $\xi=w_{1}(p)=w_{1}\left(p^{\#}\right)$, so the double covering of $\widetilde{E}_{O(2)}$ defined by the $T$-dual is the same as the original $\widetilde{E}_{S O(2)} \rightarrow \widetilde{E}_{O(2)}$.

(3) $p_{!}(h)=c_{1}(\widehat{p})$ and $(\widehat{p})_{!}\left(h^{\#}\right)=c_{1}(p)$ in $H_{O(2)}^{2}\left(\widetilde{E}, \mathbb{Z}_{\xi}\right)$.

(4) $h$ and $h^{\#}$ agree after pull-back to the correspondence space $Y \times E O(2)$ $\simeq Y$.

Therefore we conclude, assuming for simplicity that $E$ is compact and using Corollary 4.8 and the appendix in [22], that

$$
K^{\bullet}(E, h) \cong R K^{\bullet+1}\left(\widetilde{Y}_{O(2)}, h^{\#}\right) \cong K_{O(2)}^{\bullet+1}\left(\widetilde{Y}, h^{\#}\right)
$$

where $R K^{\bullet}\left(\widetilde{Y}_{O(2)}, h^{\#}\right)$ denotes the representable twisted K-theory of the compactly generated space $\widetilde{Y}_{O(2)}$ and $K_{O(2)}^{\bullet+1}\left(\widetilde{Y}, h^{\#}\right)^{\uparrow}$ denotes the $I(O(2))$-adic completion of the twisted equivariant K-theory $K_{O(2)}^{\bullet+1}\left(\widetilde{Y}, h^{\#}\right)$. Here $I(O(2))$ denotes the augmentation ideal of the representation ring $K_{O(2)}^{0}(\mathrm{pt})=$ $R(O(2))$.

\section{Acknowledgements}

VM was partially supported by the Australian Research Council Grant DP110100072. JR was partially supported by US National Science Foundation Grants DMS-0805003 and DMS-1206159, and also thanks the Department of Pure Mathematics at The University of Adelaide for its hospitality during a visit in March 2012. The authors thank the referee for useful suggestions.

\section{References}

[1] E. Alvarez, L. Alvarez-Gaumé, J. L. F. Barbón and Y. Lozano, Some global aspects of duality in string theory. Nuclear Phys. B, 415(1):71100, 1994, arXiv:hep-th/9309039.

[2] David Baraglia, Topological T-duality for general circle bundles. Pure Appl. Math. Q., 10(3):367-438, 2014, arXiv:1105.0290.

[3] David Baraglia, Topological T-duality for torus bundles with monodromy. preprint, 2012, arXiv:1201.1731. 
[4] David Baraglia, Topological T-duality with monodromy. String-Math 2011, volume 85 of Proc. Sympos. Pure Math., pages 293-302. Amer. Math. Soc., Providence, RI, 2012.

[5] Bruce Blackadar, K-theory for operator algebras, volume 5 of Mathematical Sciences Research Institute Publications. Cambridge University Press, Cambridge, second edition, 1998.

[6] Peter Bouwknegt, Jarah Evslin and Varghese Mathai, T-duality: topology change from H-flux. Comm. Math. Phys., 249(2):383-415, 2004, arXiv:hep-th/0306062.

[7] Peter Bouwknegt, Jarah Evslin and Varghese Mathai, Topology and H-flux of T-dual manifolds. Phys. Rev. Lett., 92(18):181601, 3, 2004, arXiv:hep-th/0312052.

[8] Ulrich Bunke and Thomas Schick, On the topology of T-duality. Rev. Math. Phys., 17(1):77-112, 2005, arXiv:math.GT/0405132.

[9] Ulrich Bunke, Thomas Schick and Markus Spitzweck, Periodic twisted cohomology and T-duality. Astérisque, (337):vi+134, 2011, arXiv:0805.1459.

[10] A. Connes, An analogue of the Thom isomorphism for crossed products of a $C^{*}$-algebra by an action of $\mathbb{R}$. Adv. in Math., 39(1):31-55, 1981.

[11] David Crocker, Alexander Kumjian, Iain Raeburn and Dana P. Williams, An equivariant Brauer group and actions of groups on $C^{*}$ algebras. J. Funct. Anal., 146(1):151-184, 1997.

[12] David Crocker, Iain Raeburn and Dana P. Williams, Equivariant Brauer and Picard groups and a Chase-Harrison-Rosenberg exact sequence. J. Algebra, 307(1):397-408, 2007.

[13] Calder Daenzer, A groupoid approach to noncommutative T-duality. Comm. Math. Phys., 288(1):55-96, 2009, arXiv:0704.2592.

[14] Siegfried Echterhoff, Ryszard Nest and Hervé Oyono-Oyono, Fibrations with noncommutative fibers. J. Noncommut. Geom., 3(3):377-417, 2009, arXiv:0810.0118.

[15] Siegfried Echterhoff, Ryszard Nest and Hervé Oyono-Oyono, Principal non-commutative torus bundles. Proc. Lond. Math. Soc. (3), 99(1):131, 2009, arXiv:0810.0111. 
[16] Siegfried Echterhoff and Oliver Pfante, Equivariant K-theory of finite dimensional real vector spaces. Münster J. Math., 2:65-94, 2009, arXiv:0903.1035.

[17] Victor Guillemin, Viktor Ginzburg and Yael Karshon, Moment maps, cobordisms, and Hamiltonian group actions, volume 98 of Mathematical Surveys and Monographs. American Mathematical Society, Providence, RI, 2002. Appendix J by Maxim Braverman.

[18] Masaki Izumi, Finite group actions on $C^{*}$-algebras with the Rohlin property. I. Duke Math. J., 122(2):233-280, 2004.

[19] Pierre Julg, K-théorie équivariante et produits croisés. C. R. Acad. Sci. Paris Sér. I Math., 292(13):629-632, 1981.

[20] Varghese Mathai and Jonathan Rosenberg, T-duality for torus bundles with $H$-fluxes via noncommutative topology. Comm. Math. Phys., 253(3):705-721, 2005, arXiv:hep-th/0401168.

[21] Varghese Mathai and Jonathan Rosenberg, T-duality for torus bundles with $H$-fluxes via noncommutative topology. II. The high-dimensional case and the T-duality group. Adv. Theor. Math. Phys., 10(1):123-158, 2006, arXiv:hep-th/0508084.

[22] Varghese Mathai and Siye Wu, Topology and flux of T-dual manifolds with circle actions. Comm. Math. Phys., 316(1):279-286, 2012, arXiv:1108.5045.

[23] J. P. May, Equivariant homotopy and cohomology theory, volume 91 of CBMS Regional Conference Series in Mathematics. Published for the Conference Board of the Mathematical Sciences, Washington, DC, 1996, available at http://www.math.uchicago.edu/ may/BOOKS/. With contributions by M. Cole, G. Comezaña, S. Costenoble, A. D. Elmendorf, J. P. C. Greenlees, L. G. Lewis, Jr., R. J. Piacenza, G. Triantafillou and S. Waner.

[24] Calvin C. Moore, Group extensions and cohomology for locally compact groups. III. Trans. Amer. Math. Soc., 221(1):1-33, 1976.

[25] N. Christopher Phillips, Equivariant K-theory and freeness of group actions on $C^{*}$-algebras, volume 1274 of Lecture Notes in Mathematics. Springer-Verlag, Berlin, 1987.

[26] Iain Raeburn and Jonathan Rosenberg, Crossed products of continuoustrace $C^{*}$-algebras by smooth actions. Trans. Amer. Math. Soc., 305(1):1-45, 1988. 
[27] Iain Raeburn and Dana P. Williams, Pull-backs of $C^{*}$-algebras and crossed products by certain diagonal actions. Trans. Amer. Math. Soc., 287:755-777, 1985.

[28] Jonathan Rosenberg, Topology, $C^{*}$-algebras, and string duality, volume 111 of CBMS Regional Conference Series in Mathematics. Published for the Conference Board of the Mathematical Sciences, Washington, DC, 2009.

[29] Jonathan Rosenberg, The Künneth Theorem in equivariant K-theory for actions of a cyclic group of order 2. Algebr. Geom. Topol., 13(2):1225-1241, 2013, arXiv:1208.6355.

[30] Andrew Strominger, Shing-Tung Yau and Eric Zaslow, Mirror symmetry is T-duality. Nuclear Phys. B, 479(1-2):243-259, 1996, arXiv: hep-th/9606040.

[31] David Wigner, Algebraic cohomology of topological groups. Trans. Amer. Math. Soc., 178:83-93, 1973.

Department of Pure Mathematics, University of Adelaide

Adelaide, SA 5005, Australia

E-mail address: mathai.varghese@adelaide.edu.au

Department of Mathematics, University of Maryland

College PARK, MD 20742, USA

E-mail address: jmr@math.umd.edu 